OPEN ACCESS

Edited by:

Ryoji Yanai,

Yamaguchi University, Japan

Reviewed by:

Ali Osman Saatci,

Dokuz Eylül University, Turkey

Lilian Julian

Cleveland Clinic Abu Dhabi,

United Arab Emirates

*Correspondence:

Yosuke Harada

yharada@hiroshima-u.ac.jp

Specialty section:

This article was submitted to

Ophthalmology

a section of the journal

Frontiers in Medicine

Received: 24 June 2021 Accepted: 07 December 2021

Published: 06 January 2022

Citation:

Hiyama T, Harada Y and Kiuchi Y

(2022) Clinical Characteristics and

Efficacy of Adalimumab and Low-Dose Methotrexate Combination

Therapy in Patients With

Vogt-Koyanagi-Harada Disease.

Front. Med. 8:730215.

doi: 10.3389/fmed.2021.730215

\section{Clinical Characteristics and Efficacy of Adalimumab and Low-Dose Methotrexate Combination Therapy in Patients With Vogt-Koyanagi-Harada Disease}

\author{
Tomona Hiyama, Yosuke Harada * and Yoshiaki Kiuchi
}

Department of Ophthalmology and Visual Science, Graduate School of Biomedical Sciences, Hiroshima University, Hiroshima, Japan

This retrospective study investigated the clinical characteristics and efficacy of adalimumab and low-dose methotrexate combination therapy in patients with Vogt-Koyanagi-Harada disease who were treated at Hiroshima University from February 2012 to May 2021. The patients' demographics, clinical features at administration of immunosuppressive therapy, steroid-sparing immunosuppressive therapy, side effects, and relapses were recorded. The efficacies of steroid-sparing immunosuppressive therapy (methotrexate, cyclosporine A, adalimumab, and adalimumab and methotrexate combination therapy) were analyzed. Among 62 patients, the median age at diagnosis was 47 years and the median duration of uveitis was 51 months. Systemic corticosteroid therapy was administered to $93.5 \%$ of patients $(n=58)$. Thirty-four patients $(54.8 \%)$ were treated with steroid-sparing immunosuppressive therapy. Methotrexate and cyclosporine A were administered to 12 and 22 patients, respectively; relapse occurred in 50.0\% and $22.7 \%$ of the patients, respectively. Discontinuation of cyclosporine A was required in $63.6 \%$ of patients because of side effects. Adalimumab was administered to 14 patients. Recurrence occurred in 11 patients, requiring methotrexate concomitantly. The mean dose of methotrexate at inflammatory quiescence after side effect-related dose decrease was $8.0 \mathrm{mg} /$ week $(0.13 \mathrm{mg} / \mathrm{kg})$. The median duration of combination therapy without recurrence was 20 months. There were no serious adverse events during adalimumab therapy. A high relapse rate was observed in patients receiving methotrexate; a high rate of side effects requiring discontinuation was observed in patients receiving Cyclosporine A. Patients with late-stage Vogt-Koyanagi-Harada disease may achieve better control with adalimumab and methotrexate combination therapy.

Keywords: Vogt-Koyanagi-Harada disease, uveitis, adalimumab, methotrexate, cyclosporine A, tumor necrosis factor-alpha 


\section{INTRODUCTION}

Vogt-Koyanagi-Harada disease (VKH) is an autoimmune bilateral panuveitis often associated with neurological and cutaneous involvement (1). In the early-stage of disease, granulomatous choroiditis occurs with exudative retinal detachment and optic disc swelling, causing a bilateral reduction of visual acuity $(2,3)$. Late-stage disease occurs in patients with insufficient treatment; this phase is characterized by depigmentation signs such as "sunset glow fundus" and recurrent uveitis, with an increased risk of ocular complications (1-4). Early and aggressive systemic corticosteroid therapy is the basic treatment; however, systemic corticosteroid therapy alone cannot prevent the onset of late-stage disease $(1,5)$. The early administration of additional steroid-sparing immunosuppressive therapy within 2-3 weeks of onset was reported to prevent the onset of late-stage VKH (6). Recently, mycophenolate mofetil and mycophenolic acid were found to prevent the onset of chronic VKH (7). Unfortunately, the choice of immunosuppressive therapy depends on drug availability and local regulations-in Japan, only cyclosporine A (CsA) and adalimumab (ADA) are approved for the treatment of non-infectious uveitis. VKH is the second most diagnosed intraocular inflammatory disease after sarcoidosis in Japan; many affected patients are treated solely with systemic corticosteroids at general ophthalmology hospitals. Although vigorous early immunosuppressive therapy is recommended to prevent the onset of late-stage $\mathrm{VKH}$, it is not always possible in clinical practice because of treatment availability and cost. Furthermore, it is challenging to control ocular inflammation in patients with late-stage VKH (8). In this study, we analyzed the clinical characteristics and treatment efficacies of immunosuppressive therapies in patients with VKH and focused on the treatment of patients with late-stage $\mathrm{VKH}$.

\section{MATERIALS AND METHODS}

This retrospective study reviewed data from patients with VKH who were treated at Hiroshima University Hospital (Hiroshima, Japan) from February 2012 to May 2021. The study was performed with approval from the University of Hiroshima Institutional Review Board. Written informed consent was obtained from all patients. Permission was granted by the Evaluation Committee on Unapproved or Off-labeled Drugs with High Medical Needs at Hiroshima University for methotrexate (MTX) for patients with non-infectious uveitis.

$\mathrm{VKH}$ was diagnosed in accordance with international diagnostic criteria (9). Patients were initially treated with oral corticosteroids or intravenous pulse corticosteroid therapy (1,000 mg methylprednisolone per day for 3 consecutive days) followed by the tapering of oral corticosteroids. Topical treatments were used if necessary. The study included all patients who were followed up for more than 6 months. Patients were excluded if they had an unknown date of onset or were lost to follow-up during treatment. Early-stage and late-stage VKH were diagnosed according to the classification criteria for $\mathrm{VKH}$ by the standardization of uveitis nomenclature working group (3).
The data collected in this study included patient age at diagnosis, sex, duration of uveitis, initial treatment, use of steroid-sparing immunosuppressive therapy, time until the first immunosuppressive therapy, dose of oral corticosteroids at baseline, ocular findings at baseline (anterior chamber cells $\geq 1+$, posterior synechiae, exudative retinal detachment, choroidal thickening/folding, sunset glow fundus, chorioretinal atrophy, choroidal neovascularization), neurological (headache/tinnitus/dysacusis/meningismus/cerebrospinal fluid pleocytosis)/cutaneous (vitiligo, poliosis, alopecia) features at baseline, best-corrected visual acuity at baseline, relapse during the immunosuppressive therapy, and side effects of immunosuppressive therapy. Inflammatory relapse was defined as the presence of the following: anterior chamber cells $\geq 1+$ (according to Standardization of Uveitis Nomenclature Working Group criteria), increase in subfoveal choroidal thickness or choroidal folding based on enhanced depth imaging optical coherence tomography, and indocyanine green angiography signs (e.g., choroidal vasculitis and hypofluorescent dark dots representing choroidal granulomas) (10). For patients treated with ADA and MTX combination therapy, data were obtained including previous immunosuppressive therapy, duration from onset of VKH to ADA administration, duration from ADA administration to MTX administration, dose of concomitant MTX, relapse after the combination therapy, and relapse-free period under the combination therapy.

Prior to the administration of immunosuppressive therapies (e.g., MTX, CsA, and ADA), an extensive workup was performed including complete blood count, hepatic and renal function tests, exclusion of tuberculosis and syphilis, hepatitis B and C serology, serum angiotensin converting enzyme, and chest radiography. Laboratory tests and chest radiography were performed regularly during immunosuppressive therapy. CsA blood concentration was monitored regularly. Electrocardiograms were routinely performed before starting ADA for screening heart disease $(11,12)$. An initial dose of $80 \mathrm{mg}$ ADA was administered subcutaneously, followed by $40 \mathrm{mg}$ ADA subcutaneously at 2 -week intervals, beginning 1 week after the initial dose. All patients were examined by several ophthalmologists at each visit at intervals of $\leq 2$ months. ADA was initiated when conventional therapy (i.e., oral corticosteroids $>5 \mathrm{mg}$, with or without oral immunosuppressants) failed to control ocular inflammation or when patients could not tolerate corticosteroids (e.g., in patients with diabetes mellitus or steroid-induced glaucoma) or other oral immunosuppressants (MTX or CsA). MTX or CsA were discontinued at administration of ADA. On the basis of factors such as age, severity of ocular inflammation, and range of ocular complications, some patients received ADA as a first-line steroid-sparing immunosuppressive therapy. Concomitant MTX was administered when inflammatory relapse occurred in patients who were treated with ADA.

Statistical analysis was performed using Microsoft Excel (Microsoft Corporation, Redmond, WA, USA) and JMP Pro, version 15 (SAS, Cary, NC, USA). Quantitative variables were summarized using counts (percentages), as well as means and medians (ranges). Qualitative variables were analyzed using 
TABLE 1 | Demographics of patients with Vogt-Koyanagi-Harada disease.

\begin{tabular}{lcc}
\hline Characteristics & $\boldsymbol{n}$ & \% \\
\hline Patients & 62 & 36.8 \\
Male & 25 & 54.4 \\
Female & 37 & \\
Age at diagnosis, median in years & $47(18-77)$ & \\
(range) & $51(15-148)$ & \\
Duration of uveitis, median in months & & 93.5 \\
(range) & 58 & 6.5 \\
Initial treatment of Vogt-Koyanagi-Harada disease onset & \\
$\quad$ Pulse corticosteroid therapy & 4 & \\
$\quad$ Oral corticosteroid thearpy & & \\
\end{tabular}

Fisher's exact test. Differences with $P$-values $<0.05$ were considered statistically significant.

\section{RESULTS}

Overall, 62 patients (25 men, 37 women) with VKH were included in the study. The patient demographics are summarized in Table 1. The median age at diagnosis was 47 years (range, 18-77 years). The duration of uveitis was 51 months (range, 15148 months). Systemic corticosteroid therapy was administered in $93.5 \%$ of the patients $(n=58)$. Thirty-four patients $(54.8 \%)$ were treated with steroid-sparing immunosuppressive therapy, 10 were administered treatment at the early-stage of the disease, and 24 were administered treatment at the late-stage of disease.

The baseline clinical characteristics of patients with early-stage $(n=10)$ and late-stage $(n=24) \mathrm{VKH}$ at immunosuppressive therapy administration are summarized in Table 2. The median dose of oral corticosteroids at baseline was $37.5 \mathrm{mg}$ and $11.3 \mathrm{mg}$ in early- and late-stage $\mathrm{VKH}$, respectively. The most common first choice immunosuppressive therapy was CsA in both groups. Anterior chamber cells $>1+$ were present in more than $50 \%$ of patients in both groups. Exudative retinal detachment was present in $80.0 \%$ of the patients with early-stage $\mathrm{VKH}$. At baseline, choroidal thickening/folding were observed in $\sim 80 \%$ of the patients in the early- and late-stage VKH groups. All patients with late-stage $\mathrm{VKH}$ presented with sunset glow fundus. Best-corrected visual acuity was better than 20/50 in more than $80 \%$ of the eyes in both groups.

Table 3 shows the details of immunosuppressive therapy administered to patients with VKH disease. MTX was administered to 12 patients; 11 of whom were administered MTX as a second choice. Relapse during MTX treatment occurred in $50 \%(n=6)$ of the patients. MTX was discontinued in $25.0 \%(n=3)$ of the patients because of side effects (alopecia, myelosuppression, nephrotoxicity). Of 22 patients who received CsA, 21 were administered it as a first choice. Relapse occurred in $22.7 \%(n=5)$ of the patients. The discontinuation of CsA because of side effects occurred in $63.6 \%(n=14)$ of the patients (nephrotoxicity, myelosuppression, hepatotoxicity, hematuria, edema, numbness, fever); this rate of side effects was higher than that among patients who received MTX (Fisher's exact test, $P=$ 0.0354). The median dose of CsA at which side effects occurred
TABLE 2 | Baseline clinical characteristics of patients with Early-Stage and Late-Stage Vogt-Koyanagi-Harada disease at time of administration of immunosuppressive therapy.

\begin{tabular}{|c|c|c|}
\hline Clinical characteristics & $\begin{array}{l}\text { Early-stage } \\
\qquad n=10\end{array}$ & $\begin{array}{l}\text { Late-stage } \\
\qquad n=24\end{array}$ \\
\hline $\begin{array}{l}\text { Duration from onset to first } \\
\text { immunosuppressive therapy, } \\
\text { Median in months (range) }\end{array}$ & $0.5(0-2)$ & $8(0-105)$ \\
\hline $\begin{array}{l}\text { Dose of oral corticosteroids at } \\
\text { baseline, Median in milligram (range) }\end{array}$ & $37.5(20-50)$ & $11.3(0-60)$ \\
\hline \multicolumn{3}{|l|}{ First choice immunosuppressive therapy } \\
\hline Methotrexate & 1 & 4 \\
\hline Cyclosporine A & 8 & 13 \\
\hline Adalimumab & 1 & 7 \\
\hline \multicolumn{3}{|l|}{ Ocular findings at baseline } \\
\hline Anterior chamber cell (\%) & $7(70.0)$ & $14(58.3)$ \\
\hline Posterior synechiae (\%) & $1(10.0)$ & $2(8.3)$ \\
\hline Exudative retinal detachment (\%) & $8(80.0)$ & $4(16.7)$ \\
\hline Choroidal thickening/folding (\%) & $8(80.0)$ & $19(79.2)$ \\
\hline Sunset glow fundus (\%) & $0(0)$ & $24(100)$ \\
\hline Chorioretinal atrophy (\%) & $0(0)$ & $6(25.0)$ \\
\hline Choroidal neovascularization (\%) & $0(0)$ & $2(8.3)$ \\
\hline $\begin{array}{l}\text { Neurological }{ }^{a} / \text { Cutaneous }^{b} \text { features } \\
\text { at baseline (\%) }\end{array}$ & $2(20.0)$ & $7(29.2)$ \\
\hline $\begin{array}{l}\text { Best corrected visual acuity at } \\
\text { baseline }\end{array}$ & 20 eyes & 48 eyes \\
\hline Better than 20/50 (\%) & $16(80.0)$ & $44(91.7)$ \\
\hline $20 / 50$ to $20 / 200(\%)$ & $3(15.0)$ & $3(6.3)$ \\
\hline Worse than 20/200 (\%) & $1(5.0)$ & $1(2.1)$ \\
\hline
\end{tabular}

${ }^{a}$ Headache/tinnitus/dysacusis/meningismus/cerebrospinal fluid pleocytosis.

${ }^{b}$ Vitiligo/poliosis/alopecia.

was $137.5 \mathrm{mg} /$ day $(2.3 \mathrm{mg} / \mathrm{kg})$. ADA was administered to 14 patients; as the first choice in 8 patients, as the second choice in 4 patients, and as the third choice in 2 patients. Of 14 patients, 13 were administered ADA at late-stage disease. Relapse during ADA occurred in 11 patients, who then required concomitant MTX. No side effects were observed during ADA therapy.

The 11 patients treated with ADA and MTX combination therapy following relapse during ADA monotherapy are summarized in Table 4. All 11 patients were administered $\mathrm{ADA}$ at late-stage disease. The median duration from onset to ADA administration was 5 months (range, 0-105 months). Nine out of 11 patients relapsed within the first 6 months after starting ADA, and received concomitant MTX. All 11 patients had received MTX by 12 months (median, 2 months; range, 0-12 months). The mean maximum dose of concomitant MTX was $11.5 \mathrm{mg} /$ week $(0.18 \mathrm{mg} / \mathrm{kg})$. A dose adjustment of MTX was required in five patients $(45.5 \%)$ because of side effects (hepatotoxicity and fatigue). The mean dose of MTX at inflammatory quiescence after dose adjustment was $8.0 \mathrm{mg} /$ week $(0.13 \mathrm{mg} / \mathrm{kg})$. Inflammatory relapse occurred in three patients (27.3\%) after 4, 6, and 12 months of combination therapy (patients 6, 10, and 11): subsequently, the MTX dose was increased in these three patients. The median duration of ADA and MTX combination therapy without relapse was 20 months (range, 4-31). 
TABLE 3 | Immunosuppressive therapy in patients with Vogt-Koyanagi-Harada disease.

\begin{tabular}{|c|c|c|}
\hline & $n$ & $\%$ \\
\hline \multicolumn{3}{|l|}{ Methotrexate $(n=12)$} \\
\hline First choice & 1 & 8.3 \\
\hline Second choice & 11 & 91.7 \\
\hline Early-stage & 5 & 41.7 \\
\hline Late-stage & 7 & 58.3 \\
\hline Relapse during methotrexate therapy & 6 & 50.0 \\
\hline Discontinuation due to side effects & 3 & 25.0 \\
\hline \multicolumn{3}{|l|}{ Cyclosporine A ( $n=22)$} \\
\hline First choice & 21 & 95.5 \\
\hline Second choice & 1 & 4.5 \\
\hline Early-stage & 8 & 36.4 \\
\hline Late-stage & 14 & 63.6 \\
\hline Relapse during cyclosporine A therapy & 5 & 22.7 \\
\hline Discontinuation due to side effects & 14 & 63.6 \\
\hline \multicolumn{3}{|l|}{ Adalimumab $(n=14)$} \\
\hline First choice & 8 & 57.1 \\
\hline Second choice & 4 & 28.6 \\
\hline Third choice & 2 & 14.3 \\
\hline Early-stage & 1 & 7.1 \\
\hline Late-stage & 13 & 92.9 \\
\hline Relapse during Adalimumab therapy & 11 & 78.6 \\
\hline Discontinuation due to side effects & 0 & 0.0 \\
\hline Monotherapy & 3 & 21.4 \\
\hline Adalimumab and Methotrexate combination therapy & 11 & 78.6 \\
\hline
\end{tabular}

There were no serious adverse events associated with ADA treatment in this study. Side effects occurred in 11 out of 21 patients (52.4\%) who received MTX (including MTX monotherapy and combination therapy). Side effects were managed by dose adjustment in 8 out of 21 patients $(38.1 \%)$, and, $14.3 \%(n=3)$ discontinued MTX. In contrast, side effects of CsA that required discontinuation were observed in $63.6 \%$ of the patients; nephrotoxicity was the most common side effect.

\section{DISCUSSION}

Recently, combinations of steroidal and steroid-sparing immunosuppressive therapies for $\mathrm{VKH}$ were proposed to prevent chronic recurrent disease (6). In the present study, we focused on the clinical characteristics and treatment efficacies of MTX and CsA in patients with VKH, as well as the use of ADA and low-dose MTX combination therapy for the treatment of late-stage VKH in Japanese patients.

In this study, $54.8 \%$ of all patients with $\mathrm{VKH}$ were treated with steroid-sparing immunosuppressive therapies, including MTX, CsA, and ADA. In many instances, CsA was chosen first, as this is approved for the treatment of non-infectious uveitis. Patients with early-stage $\mathrm{VKH}$ received immunosuppressive therapy approximately within 1 month of diagnosis, during the tapering of oral corticosteroids. Anterior chamber cells and exudative retinal detachment were more prominent in patients with early-stage $\mathrm{VKH}$, whereas choroidal thickening/folding was present in patients with early- and late-stage $\mathrm{VKH}$ at baseline when they received immunosuppressive therapy. Sunset glow fundus, a characteristic depigmentation sign of $\mathrm{VKH}$, was present in all patients with late-stage VKH. Neurological and cutaneous features were slightly less prominent in patients who received immunosuppressive therapy at the early-stage of disease, which may have been improved because of the aggressive corticosteroid therapy. Inflammatory relapse occurred in $50.0 \%$ and $22.7 \%$ of patients treated with MTX or CsA, respectively. Notably, the rate of side effects requiring discontinuation of medication was higher in patients treated with CsA than in patients treated with MTX. In Japan, the approved maximum dose of MTX is $16 \mathrm{mg} /$ week because of the increased prevalence of adverse events in Japanese patients (13). The higher recurrence rate in the MTX group may be associated with the insufficient MTX dose, which had been adjusted because of side effects. Furthermore, regardless of the concurrent initiation of MTX with systemic corticosteroid treatment, its effects were not apparent until a few months after administration, unlike immediate-acting agents such as CsA and ADA (14). Thus, the use of delayed-acting agents such as MTX alone may be inappropriate to prevent ongoing subclinical choroidal inflammation in patients with early-stage VKH.

As we previously suggested, VKH may require stronger antiinflammatory treatment, compared with other uveitis etiologies (15). Adalimumab is more effective when used in combination with disease-modifying antirheumatic drugs (e.g., MTX) than when used alone (16-18). In the current study, 13 out of 14 patients who were treated with $\mathrm{ADA}$ received the treatment at late-stage. Of these 13 patients, relapse occurred in 11, which required ADA and MTX combination therapy. After the onset of late-stage $\mathrm{VKH}$, the immunosuppressive effect of ADA alone might unfortunately be insufficient to control ocular flares and to prevent further functional and morphological dysfunctions. In this study, ADA and MTX combination therapy was successful in $72.7 \%$ of patients with late-stage $\mathrm{VKH}$; the median recurrence-free period was 20 months. Previously, an adequate concentration of MTX to suppress inflammation with limited hepatotoxicity in Japanese patients with RA was achieved using a dose of 10-12 mg/week (19). Previously we reported that the median maximum dose of MTX that achieved inflammatory quiescence in patients with sclerouveitis was $16 \mathrm{mg} /$ week, and the inflammatory control of non-infectious uveitis was achieved with the median dose of $12 \mathrm{mg} /$ week $(20,21)$. In this study, the median concomitant MTX dose required at inflammatory quiescence without any side effects was $8.0 \mathrm{mg} /$ week $(0.13$ $\mathrm{mg} / \mathrm{kg}$ ), which is considered low-dose therapy. This suggests that combination therapy involving ADA and MTX (at a dose lower than generally considered adequate for monotherapy) may be sufficient to achieve inflammatory control in patients in latestage VKH. In our study, $81.8 \%$ of patients who were treated with the combination therapy required MTX within 6 months after ADA administration. Because of the slow onset of action of MTX, we presume that it is preferable to initiate MTX concomitantly with ADA in patients with late-stage VKH. To the best of our knowledge, this study includes the largest series of patients with $\mathrm{VKH}$ who were treated with ADA and MTX combination therapy. 
TABLE 4 | Patients treated with adalimumab and methotrexate combination therapy.

\begin{tabular}{|c|c|c|c|c|c|c|c|c|c|c|}
\hline Patient & Sex & $\begin{array}{l}\text { Age at } \\
\text { diagnosis } \\
\text { (years) }\end{array}$ & $\begin{array}{l}\text { Duration } \\
\text { of uveitis } \\
\text { (months) }\end{array}$ & $\begin{array}{c}\text { Previous } \\
\text { immuno } \\
\text { suppressive } \\
\text { therapy }\end{array}$ & $\begin{array}{l}\text { Duration } \\
\text { from onset } \\
\text { to ADA } \\
\text { administration } \\
\text { (months) }\end{array}$ & $\begin{array}{c}\text { Duration } \\
\text { from ADA to } \\
\text { MTX } \\
\text { administration } \\
\text { (months) }\end{array}$ & $\begin{array}{l}\text { MTX dose at } \\
\text { inflammatory } \\
\text { quiescence } \\
\text { (mg) }\end{array}$ & $\begin{array}{l}\text { Side effects } \\
\text { of MTX that } \\
\text { required } \\
\text { dose } \\
\text { adjustment }\end{array}$ & $\begin{array}{c}\text { Relapse } \\
\text { after } \\
\text { combination } \\
\text { therapy }\end{array}$ & $\begin{array}{l}\text { Combination } \\
\text { therapy without } \\
\text { relapse } \\
\text { (months) }\end{array}$ \\
\hline 1 & $\mathrm{~F}$ & 59 & 19 & + & 5 & 2 & 10 & & - & 12 \\
\hline 2 & $\mathrm{~F}$ & 56 & 56 & - & 14 & 12 & 4 & Hepatotoxicity & - & 30 \\
\hline 5 & $\mathrm{~F}$ & 29 & 141 & + & 105 & 7 & 8 & & - & 28 \\
\hline 6 & M & 50 & 57 & + & 23 & 2 & 14 & & + & 12 \\
\hline 7 & M & 37 & 36 & - & 2 & 2 & 4 & Hepatotoxicity & - & 31 \\
\hline 8 & M & 42 & 37 & - & 3 & 2 & 4 & Hepatotoxicity & - & 31 \\
\hline 9 & M & 27 & 20 & + & 8 & 0 & 4 & Hepatotoxicity & - & 16 \\
\hline 10 & M & 22 & 12 & - & 2 & 0 & 16 & & + & 6 \\
\hline
\end{tabular}

M, male; F, female; ADA, adalimumab; MTX, methotrexate.

According to a previous study conducted in Japan, $\sim 61 \%$ of patients receiving systemic corticosteroid monotherapy eventually developed sunset glow fundus $(5,14,22-25)$. Thus, if the goal of successful treatment is preventing the onset of late-stage disease, there is a risk of over-treatment in onethird of patients if steroid-sparing immunosuppressive therapy is administered at the initiation of systemic corticosteroid therapy. However, when the consequences of late-stage disease (e.g., cataract, glaucoma, contrast sensitivity, color vision deficits, and extraocular complications) are weighed against the potential side effects of steroid-sparing immunosuppressive therapy, early ADA or ADA and MTX combination may be appropriate, especially in younger patients (14).

There were several limitations in this study. First, the number of patients was limited; thus, a multicenter study in Japan is needed to determine the efficacy and the rate of side effects associated with MTX and CsA, as well as ADA and MTX combination therapy, among patients with latestage $\mathrm{VKH}$ in a broader population. A direct comparison of ADA monotherapy, and ADA and MTX combination therapy should also be conducted. Second, a longer followup period is needed because of the chronic nature of VKH. Further assessment of ADA and MTX combination therapy in patients with early- and late-stage $\mathrm{VKH}$, as well as the possibility of the suspension of therapy should be conducted. Third, the duration from VKH onset to first steroid-sparing immunosuppressive therapy varied among patients in this study. A better analysis of the treatment results of MTX and CsA might have been possible if all patients had received treatment with consistent timing. Fourth, the measurement of ADA trough levels and anti-ADA antibody levels would be useful to assess their relationship with treatment suspension. The presence of antinuclear antibodies may be a predictable consequence of sufficient anti-tumor necrosis factor-alpha blockade, which may aid the treatment suspension $(26,27)$. Last, this was a retrospective study and thus included various patients with different treatment backgrounds. A prospective study is needed to investigate the use of ADA and MTX therapy in patients with VKH.
In conclusion, this study demonstrated the clinical characteristics of $\mathrm{VKH}$ in Japanese patients; a high relapse rate was observed in patients treated with MTX and a high rate of side effects requiring discontinuation was observed in patients treated with CsA. This study showed that patients with late-stage VKH may achieve better disease control with ADA and low-dose MTX combination therapy.

\section{DATA AVAILABILITY STATEMENT}

The data that support the findings of this study are available on request from the corresponding author, Yoshiaki Kiuchi. The data are not publicly available due to their containing information that could compromise the privacy of research participants.

\section{ETHICS STATEMENT}

The studies involving human participants were reviewed and approved by University of Hiroshima Institutional Review Board. The patients/participants provided their written informed consent to participate in this study. Written informed consent was obtained from the individual(s) for the publication of any potentially identifiable data included in this article.

\section{AUTHOR CONTRIBUTIONS}

$\mathrm{TH}$ and $\mathrm{YH}$ contributed to the conception, design of the study, and organized the database. TH performed the statistical analyses and wrote the first draft of the manuscript. TH, YH, and YK contributed to the manuscript revision and reading, and approved the submitted version. All authors contributed to the article and approved the submitted version.

\section{ACKNOWLEDGMENTS}

We thank Ryan Chastain-Gross, Ph.D., and J. Ludovic Croxford, Ph.D., from Edanz (https://jp.edanz.com/ac) for editing a draft of this manuscript. 


\section{REFERENCES}

1. Moorthy RS, Inomata H, Rao NA. Vogt-Koyanagi-Harada syndrome. Surv Ophthalmol. (1995) 39:265-92. doi: 10.1016/S0039-6257(05)80105-5

2. Du L, Kijlstra A, Yang P. Vogt-Koyanagi-Harada disease: novel insights into pathophysiology, diagnosis and treatment. Prog Retinal Eye Res. (2016) 52:84-111. doi: 10.1016/j.preteyeres.2016.02.002

3. Jabs DA, Denniston AK, Dick A-DD, Dunn JP, Kramer M, Mccluskey P, et al. Classification criteria for vogt-koyanagi-harada disease. Am J Ophthalmol. (2021) 228:205-11. doi: 10.1016/j.ajo.2021.03.036

4. Yang P, Ren Y, Li B, Fang W, Meng Q, Kijlstra A. Clinical characteristics of vogt-koyanagi-harada syndrome in chinese patients. Ophthalmology. (2007) 114:606-14.e3. doi: 10.1016/j.ophtha.2006.07.040

5. Nakayama M, Keino H, Watanabe T, Okada AA. Clinical science clinical features and visual outcomes of 111 patients with new-onset acute VogtKoyanagi-Harada disease treated with pulse intravenous corticosteroids. $\mathrm{Br}$ J Ophthalmol. (2019) 103:274-8. doi: 10.1136/bjophthalmol-2017-311691

6. Herbort CPJ, Abu El Asrar AM, Takeuchi M, Pavésio CE, Couto C, Hedayatfar A, et al. Catching the therapeutic window of opportunity in early initial-onset Vogt-Koyanagi-Harada uveitis can cure the disease. Int Ophthalmol. (2019) 39:1419-25. doi: 10.1007/s10792-018-0949-4

7. Abu El-Asrar AM, Dosari M, Hemachandran S, Gikandi PW, Al-Muammar A. Mycophenolate mofetil combined with systemic corticosteroids prevents progression to chronic recurrent inflammation and development of "sunset glow fundus" in initial-onset acute uveitis associated with Vogt-KoyanagiHarada disease. Acta Ophthalmol. (2017) 95:85-90. doi: 10.1111/aos.13189

8. Sonoda K-H, Hasegawa E, Namba K, Okada AA, Ohguro N, Goto H. Epidemiology of uveitis in Japan: a 2016 retrospective nationwide survey. Jpn J Ophthalmol. (2021) 65:184-90. doi: 10.1007/s10384-020-00809-1

9. Read RW, Holland GN, Rao NA, Tabbara KF, Ohno S, Arellanes-Garcia L, et al. Revised diagnostic criteria for Vogt-Koyanagi-Harada disease: report of an international committee on nomenclature. Am J Ophthalmol. (2001) 131:647-52. doi: 10.1016/S0002-9394(01)00925-4

10. Jabs DA, Nussenblatt RB, Rosenbaum JT, Standardization of Uveitis Nomenclature (SUN) Working Group. Standardization of uveitis nomenclature for reporting clinical data. Results of the first international workshop. Am J Ophthalmol. (2005) 140:509-16. doi: 10.1016/j.ajo.2005.03.057

11. Suhler EB, Jaffe GJ, Fortin E, Lim LL, Merrill PT, Dick AD, et al. Longterm safety and efficacy of adalimumab in patients with noninfectious intermediate uveitis, posterior uveitis, or panuveitis. Ophthalmology. (2021) 128:899-909. doi: 10.1016/j.ophtha.2020.10.036

12. Burmester GR, Panaccione R, Gordon KB, McIlraith MJ, Lacerda APM. Adalimumab: long-term safety in 23458 patients from global clinical trials in rheumatoid arthritis, juvenile idiopathic arthritis, ankylosing spondylitis, psoriatic arthritis, psoriasis and Crohn's disease. Ann Rheum Dis. (2013) 72:517-24. doi: 10.1136/annrheumdis-2011-201244

13. Kameda H, Fujii T, Nakajima A, Koike R, Sagawa A, Kanbe K, et al. Japan college of rheumatology guideline for the use of methotrexate in patients with rheumatoid arthritis. Mod Rheumatol. (2019) 29:3140. doi: 10.1080/14397595.2018.1472358

14. Herbort CP, Tugal-Tutkun I, Khairallah M, Abu el Asrar AM, Pavésio CE, Soheilian M. Vogt-Koyanagi-Harada disease: recurrence rates after initialonset disease differ according to treatment modality and geographic area. Int Ophthalmol. (2021) 40:2423-23. doi: 10.1007/s10792-020-01417-1

15. Hiyama T, Harada Y, Kiuchi Y. Efficacy and safety of adalimumab therapy for the treatment of non-infectious uveitis: efficacy comparison among uveitis aetiologies. Ocul Immunol Inflamm. (2021) doi: 10.1080/09273948.2020.1857791. [Epub ahead of print].

16. Burmester GR, Kivitz AJ, Kupper H, Arulmani U, Florentinus S, Goss SL, et al. Efficacy and safety of ascending methotrexate dose in combination with adalimumab: the randomised CONCERTO trial. Ann Rheum Dis. (2015) 74:1037-44. doi: 10.1136/annrheumdis-2013-204769
17. Krieckaert CL, Nurmohamed MT, Wolbink GJ. Methotrexate reduces immunogenicity in adalimumab treated rheumatoid arthritis patients in a dose dependent manner. Ann Rheum Dis. (2012) 71:1914 LP-15. doi: 10.1136/annrheumdis-2012-201544

18. Cordero-Coma M, Calleja-Antolín S, Garzo-García I, Nuñez-Garnés AM, Álvarez-Castro C, Franco-Benito $\mathrm{M}$, et al. Adalimumab for treatment of noninfectious uveitis: immunogenicity and clinical relevance of measuring serum drug levels and antidrug antibodies. Ophthalmology. (2016) 123:261825. doi: 10.1016/j.ophtha.2016.08.025

19. Takahashi C, Kaneko Y, Okano Y, Taguchi H, Oshima H, Izumi $\mathrm{K}$, et al. Association of erythrocyte methotrexate-polyglutamate levels with the efficacy and hepatotoxicity of methotrexate in patients with rheumatoid arthritis: a 76-week prospective study. RMD Open. (2017) 3:e000363. doi: 10.1136/rmdopen-2016-000363

20. Hiyama T, Harada Y, Kiuchi Y. Clinical characteristics and efficacy of methotrexate in Japanese patients with noninfectious scleritis. Jpn J Ophthalmol. (2021) 65:97-106. doi: 10.1007/s10384-020-00778-5

21. Harada Y, Hiyama T, Kiuchi Y. Methotrexate effectively controls ocular inflammation in japanese patients with non-infectious uveitis. Front Med. (2021) 8:2194. doi: 10.3389/fmed.2021.732427

22. Nishioka $Y$, Sakamoto $M$, Kinukawa $N$, Sanui $H$, Inomata $H$, Nose Y. Recurrence risk factors in patients with the Vogt-KoyanagiHarada syndrome in Japan. Ocul Immunol Inflamm. (1995) 3:73-80. doi: 10.3109/09273949509085034

23. Keino H, Goto H, Usui M. Short communication sunset glow fundus in Vogt-Koyanagi-Harada disease with or without chronic ocular inflammation. Arch Clin Exp Ophthalmol. (2002) 240:878-82. doi: 10.1007/s00417-0020538-z

24. Iwahashi C, Kensuke O, Noriyasu H, Kei N, Ohguro N, Nishida $\mathrm{K}$. Incidence and clinical features of recurrent Vogt-KoyanagiHarada disease in Japanese individuals. Jpn J Ophthalmol. (2015) 59:157-63. doi: 10.1007/s10384-015-0377-1

25. Keino $\mathrm{H}$, Goto $\mathrm{H}$, Mori $\mathrm{H}$, Iwasaki $\mathrm{T}$, Usui $\mathrm{M}$. Association between severity of inflammation in CNS and development of sunset glow fundus in Vogt-Koyanagi-Harada disease. Am J Ophthalmol. (2006) 141:11402. doi: 10.1016/j.ajo.2006.01.017

26. Atzeni F, Turiel M, Capsoni F, Doria A, Meroni P, Sarzi-Puttinia P. Autoimmunity and Anti-TNF-a agents. Ann N Y Acad Sci. (2005) 1051:55969. doi: 10.1196/annals.1361.100

27. Lügering $A$, Schmidt $M$, Lügering $N$, Pauels $H G$, Domschke $W$, Kucharzik T. Infliximab induces apoptosis in monocytes from patients with chronic active Crohn's disease by using a caspase-dependent pathway. Gastroenterology. (2001) 121:1145-57. doi: 10.1053/gast.2001. 28702

Conflict of Interest: The authors declare that the research was conducted in the absence of any commercial or financial relationships that could be construed as a potential conflict of interest.

Publisher's Note: All claims expressed in this article are solely those of the authors and do not necessarily represent those of their affiliated organizations, or those of the publisher, the editors and the reviewers. Any product that may be evaluated in this article, or claim that may be made by its manufacturer, is not guaranteed or endorsed by the publisher.

Copyright (c) 2022 Hiyama, Harada and Kiuchi. This is an open-access article distributed under the terms of the Creative Commons Attribution License (CC BY). The use, distribution or reproduction in other forums is permitted, provided the original author(s) and the copyright owner(s) are credited and that the original publication in this journal is cited, in accordance with accepted academic practice. No use, distribution or reproduction is permitted which does not comply with these terms. 